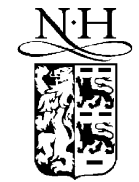

ELSEVIER

\title{
Phase explosion and its time lag in nanosecond laser ablation
}

\author{
Xianfan $\mathrm{Xu}^{*}$ \\ School of Mechanical Engineering, Purdue University, West Lafayette, IN 47907-1288, USA
}

\begin{abstract}
This work investigates interface kinetics during nanosecond pulsed excimer ablation of a metal. During laser heating, the surface can reach a temperature higher than the normal boiling point, resulting in a superheated, metastable state. Phase explosion occurs as the temperature approaches the thermodynamic critical point, which turns the melt into a mixture of liquid and vapor. However, for phase explosion, there is a certain time needed for a vapor embryo to grow to a critical nucleus, called the time lag of nucleation. This time lag becomes important in ablation induced by nanosecond or shorter pulsed lasers. This paper discusses experiments for investigating non-equilibrium phase change phenomena during nanosecond excimer laser ablation of a metal. Evidences of the metastable state in liquid and phase explosion are presented. The surface temperaturepressure relation is found to deviate from the commonly used equilibrium Clausius-Clapeyron equation. Also, for the first time, the time lag of nucleation during nanosecond laser ablation is found to be around $5 \mathrm{~ns}$.
\end{abstract}

(C) 2002 Elsevier Science B.V. All rights reserved.

Keywords: Non-equilibrium phase change; Phase explosion; Time lag; Laser ablation

\section{Introduction}

Laser ablation of a metal involves complex thermal phenomena, including rapid heating, non-equilibrium phase change, superheating, and rapid nucleation in a superheated liquid. The thermal mechanisms of pulsed laser ablation include surface normal evaporation and homogeneous boiling, or phase explosion. During high power pulsed laser ablation, phase explosion could be an important ablation mechanism [1-4], which occurs when the temperature of a superheated liquid approaches the thermodynamic critical point, and the homogeneous nucleation rate is sufficiently large to generate a large amount of nuclei in a short period of time. Experiments showed that phase explosion indeed occurred during nanosecond pulsed laser

\footnotetext{
*Tel.: +1-765-494-5639; fax: +1-765-494-0539. E-mail address: xxu@ecn.purdue.edu (X. Xu).
}

ablation of a metal [2], and surface temperaturepressure relation could deviate from the commonly used equilibrium Clausius-Clapeyron relation $[5,6]$.

This paper is focused on the non-equilibrium phase change process in a metal induced by a nanosecond pulsed excimer laser. Experiments are performed in the laser fluence range from 2.5 to $9 \mathrm{~J} / \mathrm{cm}^{2}$, which is commonly used for many applications including pulsed laser deposition and micromachining. Phase explosion induced by laser heating will be briefly described. The nucleation process and its time lag in a superheated liquid leading to phase explosion is studied.

\section{Phase explosion and its time lag}

The phase change process induced by pulsed laser heating can be best illustrated using the pressure- 


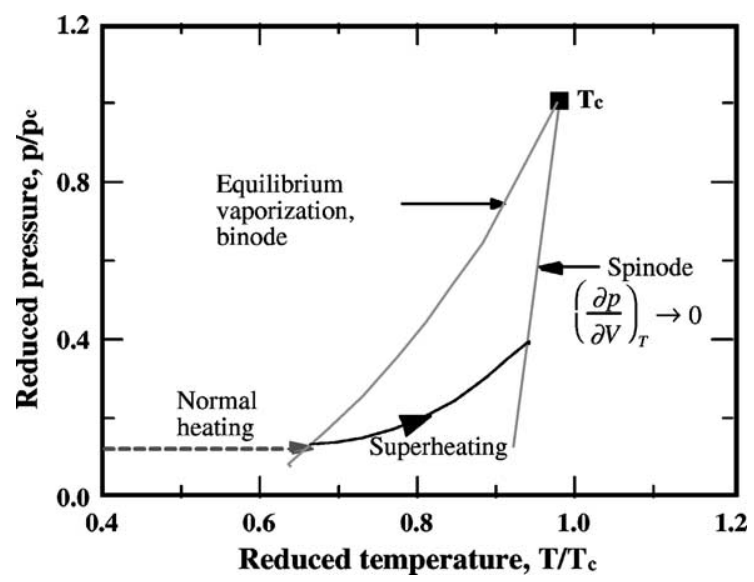

Fig. 1. The $p-T$ diagram of a liquid metal near the critical point.

temperature diagram shown in Fig. 1. The "normal heating' indicates heating of a liquid metal when the temperature is below the boiling temperature. The binode line represents equilibrium between the surface temperature $T$ and the vapor saturation pressure $p_{\mathrm{s}}$, which is calculated from the Clausius-Clapeyron equation:

$p_{\mathrm{s}}=p_{0} \exp \left\{\frac{H_{\mathrm{lv}}\left(T-T_{\mathrm{b}}\right)}{R T T_{\mathrm{b}}}\right\}$

where $p_{0}$ is the ambient pressure, $H_{1 \mathrm{v}}$ the enthalpy of vaporization, and $T_{\mathrm{b}}$ the equilibrium liquid-vapor temperature at the ambient pressure. During high power pulsed laser heating, it is possible to heat a liquid metal to temperatures above the boiling point while the surface vapor pressure is not built up as rapidly. The liquid is then superheated, i.e., its temperature is higher than the vaporization temperature corresponding to its surface pressure. In this case, the heating process follows a superheating line shown in Fig. 1, and the liquid is in a metastable state.

There is an upper limit for superheating of a liquid, the spinode [7], which can be obtained from a calculation of the nucleation rate using the Döring and Volmer's theory $[8,9]$ :

$J=N\left(\frac{3 \sigma}{\pi m}\right)^{1 / 2} \exp \left(\frac{-W_{\mathrm{cr}}}{k_{\mathrm{B}} T}\right)$

where $W_{\text {cr }}$ is the energy needed to form critical vapor nuclei at temperature $T, N$ the number of liquid molecules per unit volume, and $\sigma$ the surface tension. According to Eq. (2), the spontaneous nucleation rate increases exponentially with temperature. During pulsed laser heating, the amount of nuclei generated by spontaneous nucleation is negligible at temperatures lower than $0.9 T_{\mathrm{c}}$. At a temperature of about $0.9 T_{\mathrm{c}}$, a significant number of nuclei can be formed. Hence, explosive phase change occurs, which turns the liquid into a mixture of liquid and vapor.

One important factor in analyzing phase change induced by pulsed laser heating is the time required for a vapor embryo to grow to a critical nucleus. This time is also called the time lag for nucleation. The time lag must be considered in pulsed laser ablation since it is on the same order of the laser pulse duration. Eq. (2) can be modified to account for this time lag, $\tau$, which can be expressed as

$J=N\left(\frac{3 \sigma}{\pi m}\right)^{1 / 2} \exp \left(\frac{-W_{\mathrm{cr}}}{k_{\mathrm{B}} T}\right) \exp \left(\frac{-\tau}{t}\right)$

where $t$ is the time duration for which the liquid is superheated. The time lag $\tau$ has been estimated to be [9]

$\tau \approx\left(\frac{2 \pi M}{R T}\right)^{1 / 2} \frac{4 \pi \sigma p_{\mathrm{s}}}{\left(p_{\mathrm{s}}-p_{1}\right)^{2}}$

where $M$ is the molar weight of the substance. For metals, the time lag was estimated to be between 1 and 10 ns [9].

\section{Experimental investigation}

\subsection{Experiments and results}

A $\mathrm{KrF}$ excimer laser with a wavelength of $248 \mathrm{~nm}$ and a pulsewidth of $25 \mathrm{~ns}$ (FWHM) is used in the experiments. Transient transmissivity of laser beam through the laser-induced vapor plume, scattering of laser beam from the laser-induced vapor plume, transient location and velocity of the laser-induced vapor front, and ablation depth per laser pulse are measured. $\mathrm{Ni}$ is used as the target. Details of the experiments have been given elsewhere [3]. A summary of the results is shown in Fig. 2.

Fig. 2a shows the percentage of laser energy scattered from the vapor plume. It is seen that there is 

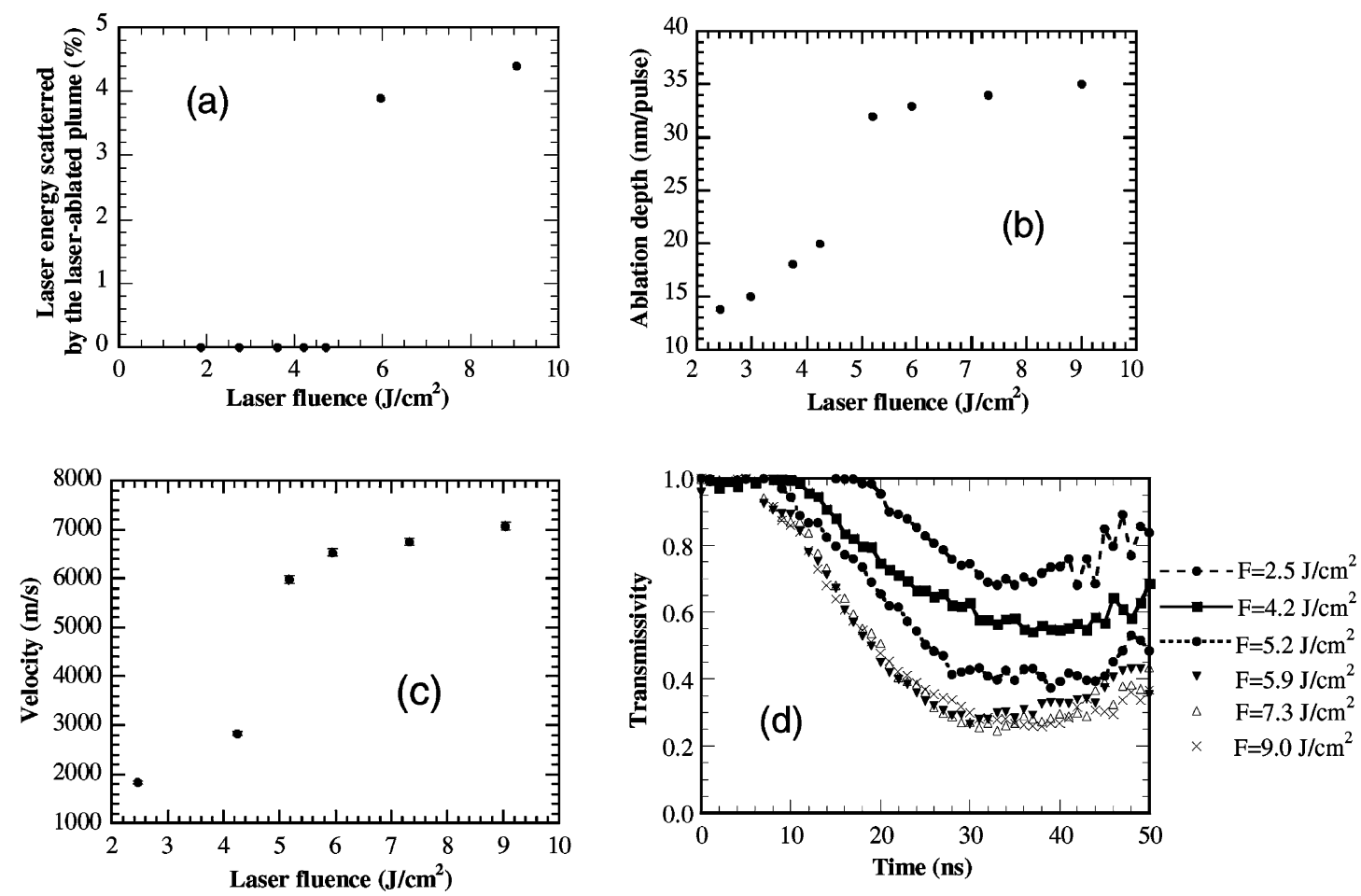

Fig. 2. (a) Percent of laser energy scattered to the ambient, (b) ablation depth, (c) vapor velocity, and (d) transient transmissivity of vapor as a function of laser fluence.

almost no scattering (less than $0.5 \%$, the measurement resolution) in the low laser fluence region. When the laser fluence is higher than $5.2 \mathrm{~J} / \mathrm{cm}^{2}$, the percentage of laser energy scattered by the plume is increased to about $4-5 \%$. Fig. 2b shows the ablation depth per laser pulse at different laser fluences. The ablation depth increases from 14 to $20 \mathrm{~nm}$ when the laser fluence is less than $4.0 \mathrm{~J} / \mathrm{cm}^{2}$. When the laser fluence increases from 4.2 to $5.2 \mathrm{~J} / \mathrm{cm}^{2}$, a jump increase in the ablation depth is observed, and stays relatively a constant at higher laser fluences. Fig. 2c shows the averaged velocity of the laser-evaporated vapor. It is seen that the vapor velocity increases with the laser fluence. A sudden jump of the velocity is seen at the laser fluence of $4.2 \mathrm{~J} / \mathrm{cm}^{2}$. In the laser fluence range between 5.2 and $9 \mathrm{~J} / \mathrm{cm}^{2}$, the velocity is almost a constant. Fig. $2 \mathrm{~d}$ shows the transient transmissivity of the vapor at different laser fluences. The transmissivity is almost identical when the laser fluence is higher than $5.2 \mathrm{~J} / \mathrm{cm}^{2}$, which is exactly the same fluence region in which the velocity of the vapor changes little.
These phenomena are explained as a result of phase explosion occurring at laser fluence around $5 \mathrm{~J} / \mathrm{cm}^{2}$. Scattering of laser energy is due to large size (on the order of sub-micron or larger) droplets in the vapor plume instead of (atomic) vapor (note that scattering is not due to the ignition of plasma, since plasma is observed at fluences lower than $5 \mathrm{~J} / \mathrm{cm}^{2}$ ). Therefore, there are no droplets in the vapor plume at low fluences, the materials removal is due to surface evaporation. This is also confirmed by collecting evaporated material using a glass slide at about $1 \mathrm{~cm}$ above the target, at laser fluences below and higher than $5 \mathrm{~J} / \mathrm{cm}^{2}$. At high fluences, droplets are generated due to phase explosion, which cause scattering. For the ablation depth, when explosive phase change occurs, the melted layer is turned into a liquid-vapor mixture. Therefore, the increase of the ablation depth at the laser fluence of $5.2 \mathrm{~J} / \mathrm{cm}^{2}$ also indicates the transition from surface evaporation to phase explosion.

The velocity of the vapor plume is determined by the pressure and the temperature at the surface. 
The constant velocity at high laser fluences indicates that the surface temperature is not affected by the increase of laser fluence. Such a constant surface temperature can be explained as a result of phase explosion. As discussed earlier, the surface temperature during phase explosion is about $0.9 T_{\mathrm{c}}$. For the transmission data, extinction of the laser beam is determined by the cross-section of the energized atoms, which in turn is determined by the temperature of the vapor plume. Thus, the transmission data also indicate the occurrence of phase explosion.

Therefore, these four independent experiments all demonstrate that surface evaporation occurs at laser fluences below $4 \mathrm{~J} / \mathrm{cm}^{2}$, and phase explosion takes place when the laser fluence is higher than about $5 \mathrm{~J} / \mathrm{cm}^{2}$.

\subsection{Interface superheating and the time lag of nucleation}

The pressure at the evaporating surface is needed to understand the evaporation kinetics, which is measured with the use of a PVDF transducer [10]. Of particular interest is the pressure when phase explosion occurs (at $5.2 \mathrm{~J} / \mathrm{cm}^{2}$ ), which is determined to be about 600 bar. Fig. 3 shows the Clausius-Clapeyron equation for $\mathrm{Ni}$, together with the experimental data point at $5.2 \mathrm{~J} / \mathrm{cm}^{2}$. It can be seen that the measured pressure is well below the equilibrium pressure, showing that the liquid is in a superheated state. The equilibrium surface temperature-pressure relation is not valid for pulsed laser ablation.

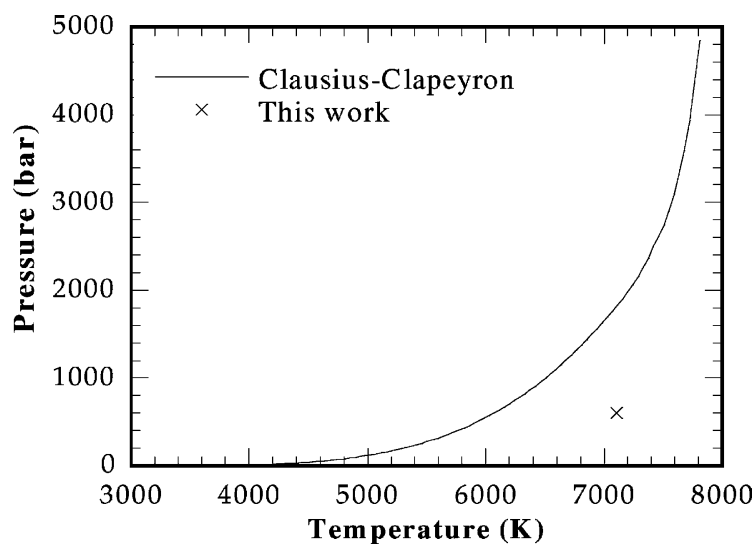

Fig. 3. Comparison between the Clausius-Clapeyron relation and the measured pressure at $0.9 T_{\mathrm{c}}$.

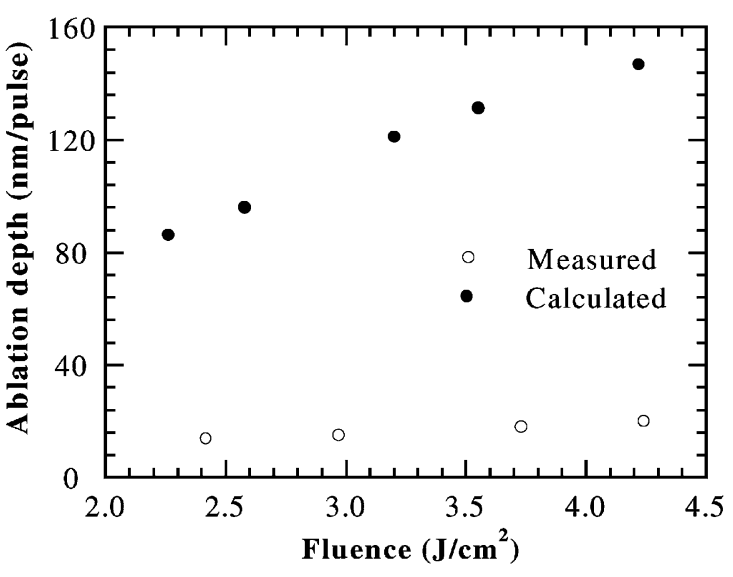

Fig. 4. Comparison between the measured ablation depth and the values calculated using transient pressure data and the equilibrium kinetic relation.

The validity of the equilibrium evaporation kinetics is also examined by computing the evaporation depth from the measured pressure using the ClausiusClapeyron equation, Eq. (1), and comparing it with the measured data. The transient surface temperature $T$ is first calculated from the measured transient surface pressure $p$ using Eq. (1). From $T$ and $p$, the evaporation velocity, $V_{\mathrm{lv}}$ can be calculated from

$V_{\mathrm{lv}}=\frac{m p_{\mathrm{s}}}{\rho_{\mathrm{l}}\left(2 \pi m k_{\mathrm{B}} T\right)^{1 / 2}}$

The ablation depth per laser pulse is then obtained by integrating the evaporation velocity over time. The calculated ablation depths are shown in Fig. 4. It can be seen that the calculated values are much larger than the measured data. This large discrepancy again indicates that the equilibrium interface kinetics is not valid during nanosecond pulsed laser evaporation.

Fig. $2 \mathrm{~d}$ reveals another phenomenon, that the onset of ablation, which can be obtained from Fig. $2 d$ as the time when transmission starts to decrease, is about the same at laser fluences higher than the threshold for phase explosion. The onset of ablation is also determined from the measured position of the vapor front vs. time (which is measured using the optical deflection technique [3] and is used to obtain the velocity of the vapor front) shown in Fig. 5. The onset of ablation obtained using these two methods is shown in Fig. 6a and $b$. Both figures show that, when the laser fluence is higher than $5.2 \mathrm{~J} / \mathrm{cm}^{2}$, the onset of ablation does not 


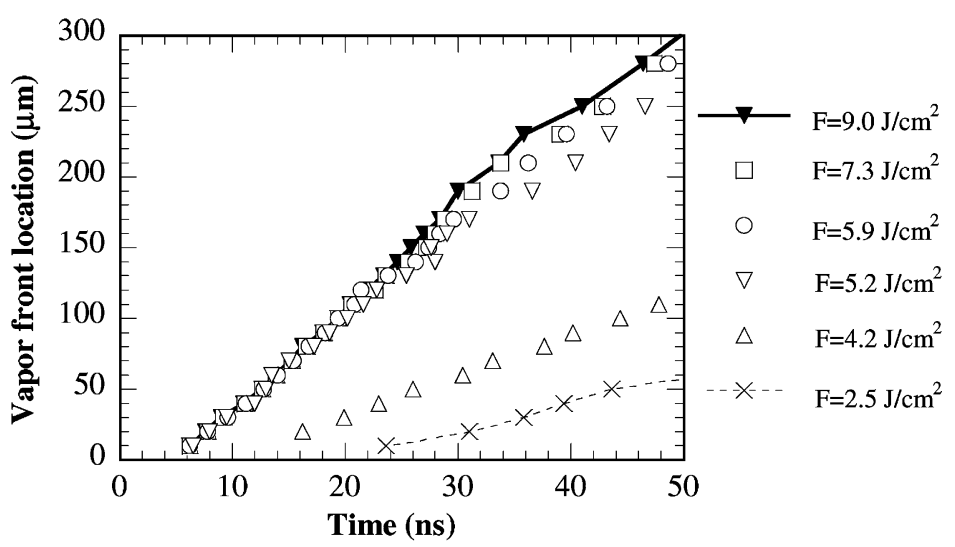

Fig. 5. Transient location of the vapor front as a function of laser fluence.
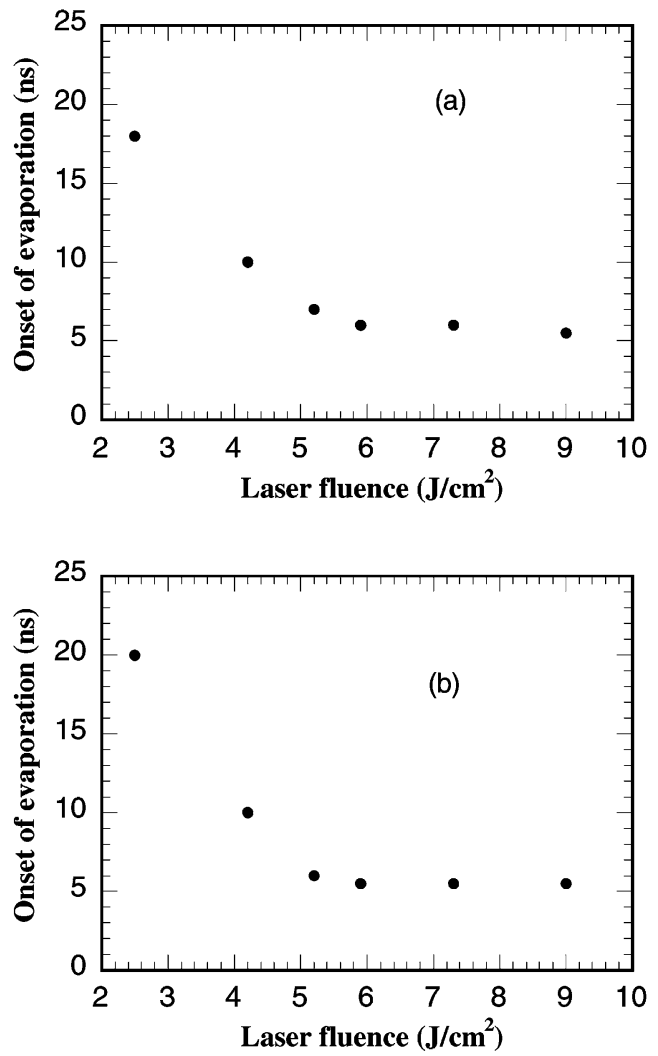

Fig. 6. Onset of evaporation as a function of laser fluence determined from (a) the transient transmissivity through the vapor, and (b) the transient location of the vapor front. change with the laser fluence, but remains at around $5.5 \mathrm{~ns}$. The two independent measurements provide almost identical results. The constant onset of ablation at laser fluences higher than $5.2 \mathrm{~J} / \mathrm{cm}^{2}$ can be explained in terms of the time lag for phase explosion. As discussed previously, before phase explosion can take place, there is a certain time needed for a vapor embryo to grow to a critical nucleus. The constant onset at laser fluences higher than the threshold of phase explosion shows that the time lag of nucleation has prevented phase explosion to occur at an earlier time when the laser fluence is increased; and this time lag, according to the experimental data, is about $5.5 \mathrm{~ns}$.

One interesting issue is what would occur if the laser pulsewidth is much shorter than the time lag of nucleation. Many experiments have shown the threshold nature of sub-nanosecond laser ablation [11,12], and phase explosion was explained as the ablation mechanisms [13]. However, given the time lag much longer than the pulsewidth, detailed studies are needed to gain a thorough understanding of ablation mechanism in a pico- or femtosecond laser ablation.

\section{Conclusions}

Non-equilibrium phase change during nanosecond pulsed excimer laser ablation of nickel was investigated. Results of experiments showed surface evaporation 
occurred at laser fluences below $4 \mathrm{~J} / \mathrm{cm}^{2}$, and phase explosion occurred at the laser fluences higher than $5 \mathrm{~J} / \mathrm{cm}^{2}$. The surface kinetic relation deviated significantly from the equilibrium Clausius-Clapeyron equation. The time lag of phase explosion during excimer laser ablation of a nickel target was found to be around $5.5 \mathrm{~ns}$.

\section{Acknowledgements}

Support of this work by the National Science Foundation and the Office of Naval Research is gratefully acknowledged.

\section{References}

[1] A. Miotello, R. Kelly, Appl. Phys. Lett. 67 (1995) 3535.

[2] K.H. Song, X. Xu, Appl. Surf. Sci. 127-129 (1998) 111.

[3] X. Xu, in: C.-L. Tien, F.P. Incropera, V. Prasad (Eds.), Annual Review of Heat Transfer, Vol. 12, 2001, pp. 79-115.
[4] D.A. Willis, X. Xu, Int. J. Heat Mass Trans., in press.

[5] X. Xu, K.H. Song, Appl. Phys. A 69 (1999) S869.

[6] X. Xu, K.H. Song, Mater. Sci. Eng. A 292 (2000) 162.

[7] W. Fucke, U. Seydel, High Temperature High Pressure 12 (1980) 419.

[8] V.P. Carey, Liquid-Vapor Phase-change Phenomena: An Introduction to the Thermophysics of Vaporization and Condensation Processes in Heat Transfer Equipment, Hemisphere, Washington, DC, 1992.

[9] V.P. Skripov, Metastable Liquids, Wiley, New York, 1974.

[10] K.H. Song, X. Xu, in: Proceedings of the 1998 ASME International Mechanical Engineering Congress and Exposition, HTD-Vol. 361-4, American Society of Mechanical Engineers, New York, 1998, p. 79.

[11] D.A. Willis, X. Xu, in: Proceedings of the 2000 International Congress on Applications of Lasers and Electro-optics, Vol. 90, Laser Institute of America, Orlando, FL, 2000, p. A36.

[12] A. Cavalleri, K. Sokolowski-Tinten, J. Bialkowski, D. von der Linde, Appl. Phys. Lett. 72 (1998) 2385.

[13] K. Sokolowski-Tinken, J. Bialkowski, M. Boing, A. Cavalleri, D. von der Linde, Bulk, in: Proceedings of the Quantum Electronics and Laser Science Conference, OSA Technical Digest, Optical Society of America, Washington, DC, 1999, p. 231. 